\title{
Dielectric Relaxation Studies of Hydrogen Bonded Complexes of Benzamide and Acetamide with Halogenated Phenols Using X-band Microwave Frequency
}

\author{
A. Aathif Basha ${ }^{1, *}$ and F. Liakath Ali Khan ${ }^{1}$ \\ ${ }^{1}$ PG and Research Department of Physics, Islamiah College (Autonomous), Vaniyambadi 635752, Tamilnadu, India \\ *Corresponding author: aatifbasha@gmail.com
}

\author{
KEYWORDS \\ Amides \\ Dielectric parameters \\ H-bonding \\ Halogenated phenols \\ Microwave bench X-band \\ SUBMITTED 27 June 2021 \\ REVISED 23 October 2021 \\ ACCEPTED 17 December2021
}

\begin{abstract}
At $308 \mathrm{~K}$, using a $9.37 \mathrm{GHz}$ dielectric relaxation setup, dielectric studies of hydrogen bonded complexes of benzamide and acetamide with 4-fluorophenol, 4-bromophenol, 4-chlorophenol, and 4-iodophenol in benzene were performed. Various dielectric parameters (such as $\epsilon^{\prime}, \epsilon^{\prime \prime}, \epsilon 0, \epsilon \infty$ ) were tested. The steric interactions of the proton donor determined the group rotation relaxation time $\tau(2)$, whereas the significance of the single frequency method of Higasi et al. for multiple relaxation time $\tau(1)$ was determined by the hydrogen bonding power of the phenolic hydrogen. The presence of a 1:1 complex system between the prepared samples, as well as a charge transfer between the free hydroxyl group of phenols and the carbonyl group of amides was confirmed by the fact that the relaxation time and molar free energy activation of the 1:1 molar ratio were greater than some other higher molar ratios (i.e. 3:1, 2:1, 1:2, 1:3).
\end{abstract}

(c) The Author(s) 2021. This article is distributed under a Creative Commons Attribution-ShareAlike 4.0 International license.

\section{INTRODUCTION}

Dielectric materials include electromagnetic properties of permittivity, permeability, and conductivity, which are the most important identifying parameters in terms of loss and lossless dielectric properties. Researchers have proposed a number of ways to measure microwave dielectric constants and dielectric losses of liquids; this method of measurement is known as the Heston-Smythe method for low loss liquids (Sisodia and Raguvanshi 2005). Dielectric properties include dielectric constant, relaxation time, and dielectric loss, which are electromagnetic properties of materials that scientists and engineers are interested in. In electromagnetic-based medical technology, electromagnetic waves are used for medical therapy and diagnostics all over the world (Gioia et al. 2018). The investigations of the relationship of two polar particles because of hydrogen holding from the dielectric relaxing estimations at microwave frequencies are exceptional. Amides are inescapable in nature, these linkages are effectively framed, present primary inflexibility, and oppose hydrolysis. Amides incorporate numerous significant organic mixtures and it has $\mathrm{N}-\mathrm{H}$ dipoles permits amides to work as H-security benefactors also (Jeffrey and Saenger 1991). Amides have been studied spectroscopically, physically, and theoretically to see how they can contain intermolecular hydrogen.

For quite some time, the effect of hydrogen keeping in the configuration of fluids and their unwinding behaviour has been studied. The discovery of hydrogen keeping contributed to the development of multimers, which are partners with a finite number of atoms. The existence and multimer motions of such fluids are often deciphered. Critical amides such acetamide and benzamide are strong pro- ton donors and acceptors, and whose intermolecular hydrogen bonds are definitely connected. As a result, they have physical characteristics that show a high dependency on the temperature and fixation of dissolvable climate. Dielectric examines have regularly end up being an integral asset to give experiences into the components of affiliation and reorientation elements of dipolar fluids (Kumar et al. 2010; Grant et al. 1978; Bone and Pethig 1985). Hydrogen bonds comprised a fascinating class of intermolecular associations which are of outrageous significance in numerous fields of science and sub-atomic science. The dielectric complexation of hydrogen-reinforced mixtures in nonpolar dissolvable gives important data in regards to atomic connection of fortified development in arrangement. The investigations of $\mathrm{H}$-obligations of the sort $\mathrm{O}=\mathrm{H}-\mathrm{O}=\mathrm{C}$ possesses a place of extensive significance by approach of that one identifies with the investigation of biopolymers. As a result, the analysis besides information on the dielectric characteristics of amide-glycol combinations in non-polar solvents is relied upon towards provide useful as well as important interaction boundaries for the appropriate planning of current premium transesterification cycles.

Because microwaves can detect weak molecule interactions, the study of dielectric relaxation of polar liquids in non-polar solvents using microwave absorption techniques provides significant information on the various types of molecular associations present in the solutions (Thakur and Sharma 2000; Vyas and Rana 2002; Rangra and Sharma 2004; Chaudhari et al. 2001; Rewar and Bhatnagar 2002). The hydrogen holding between free hydroxyl collection of phenols and carbonyl gathering of amides was studied employing a dynamic strategy in the latest research, taking into account both modern and rational needs. When a pro- 
ton giver is blended in with a proton acceptor in a nonpolar dissolvable, a dielectric unwinding analysis will provide precise details on the structure of $\mathrm{H}$-reinforced complexes. The combined solvents have been used in a variety of synthetic, organic medicine, electronic, biophysics, dense matter physical science, and research centre applications in our current study. The dielectric examination of hydrogen fortified mixtures in non-polar dissolvable gives important data in regards to sub-atomic communication of complex development in arrangements. As of late, dielectric unwinding conduct of combinations of polar particles under fluctuating states of complexation heat and climate reasons takes impressive awareness. This investigation is required to give a superior comprehension of the idea of the atomic direction measure. The dielectric unwinding practices of amides are relied upon to be like that of n-alcohols (Sengwa 1994). Existence of strong intermolecular attractions in liquid mixtures results in the formation of charge transfer or H-bonded complexes depending on the components of the system (Kumar et al. 2014).

The present study investigated the dielectric behaviour of benzamide and acetamide in the presence of halogenated phenols in benzene in order to learn more about the strong dielectric behaviour of amides in phenol arrangements. Because phenols are utilized as diluters or codiluters, they have a variety of interesting effects that are essential to organic structures.

\section{MATERIALS AND METHODS}

Aldrich assortments of benzamide and acetamide with 4-fluorophenol, 4-bromophenol, 4-chlorophenol, and 4iodophenol were utilized. The proton donors (phenols) and the acceptors (amides) were separately dissolved at the same concentration ( 0.3 moles/litre) in benzene. Their dielectric constants were measured separately. The molar proportions of the amide-phenol tertiary combinations were continually varied in the ratios of 3:1, 2:1, 1:1, 1:2, and $1: 3$, in any case maintaining the solute fixation in benzene constant.Using an X-band microwave table, the dielectric constant $\left(\epsilon^{\prime}\right)$ and dielectric loss $\left(\epsilon^{\prime \prime}\right)$ were estimated. The static dielectric constants were calculated at $308 \mathrm{~K}$ utilizing a standard equipment, the Dipole metre DM-01 from Wissenschaijftlich Technische Werksatter, Germany, which was powered by 220V (Sivagurunathan and Vijaya-

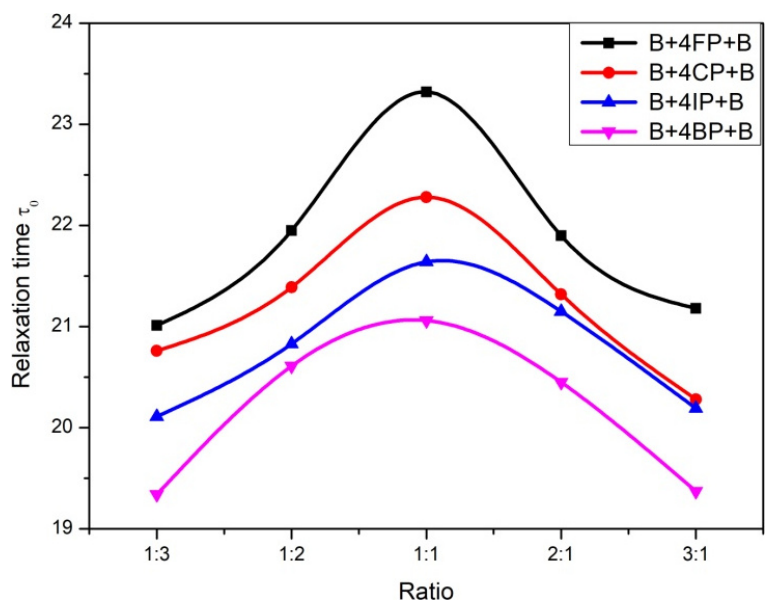

(a) balan 2010). An Abbe's refractometer was used to calculate the refractive index $\mathrm{nD}$ of all the solutions, and the measurements for $\epsilon^{\prime}$ and $\epsilon^{\prime \prime}$ are correct to \pm 1 and \pm 5 , respectively (Malathi et al. 2003). All measurements were taken at $35^{\circ} \mathrm{C}$, with the temperature being held within $0.5^{\circ} \mathrm{C}$ by a thermostat. The calculation of dielectric constants and refractive indices had uncertainties of 0.0005 and 0.0002 , respectively. The viscosities of liquids are measured using Ostwald's viscometer. A liquid cell was used to link a variable attenuator and a slotted line waveguide with a probe slit in the wide face. A ferrite isolator carried the signal from the Klystron to the attenuator. This isolator only allows power to flow freely in one direction: forward and it strongly attenuates the reverse wave. The microwave power was transmitted into the liquid via a Teflon window with negligible dielectric loss from the slotted line. The microwave power is measured by the probe, which is a crystal detector. A sensitive spot galvanometer receives the microwave current (Tabassum and Pawar 2018). Many of the chemicals used here had their physical parameters tested against their literature values.

\subsection{Dielectric parameters}

From Higasi's method (Srinivasan et al. 2003), the average relaxation time $\tau_{(1)}$ is described by Equation 1 .

$$
\tau_{(1)}=\frac{a^{\prime \prime}}{\omega\left(a^{\prime}-a_{\infty}\right)}
$$

While the whole dielectric relaxation $\tau_{(2)}$ is given by Equations 2 and 3 .

$$
\begin{gathered}
\tau_{(2)}=\frac{a_{0}-a^{\prime}}{\omega a^{\prime \prime}} \\
\tau_{(0)}=\sqrt{\tau_{(1)} \tau_{(2)}}
\end{gathered}
$$

The Debye's equation in terms of $a_{0}, a^{\prime}, a_{\infty}$ and $a$ yields Equations 4, 5, and 6 (Higasi 1966).

$$
\epsilon_{0}=\epsilon_{01}+a_{0} w_{2}
$$

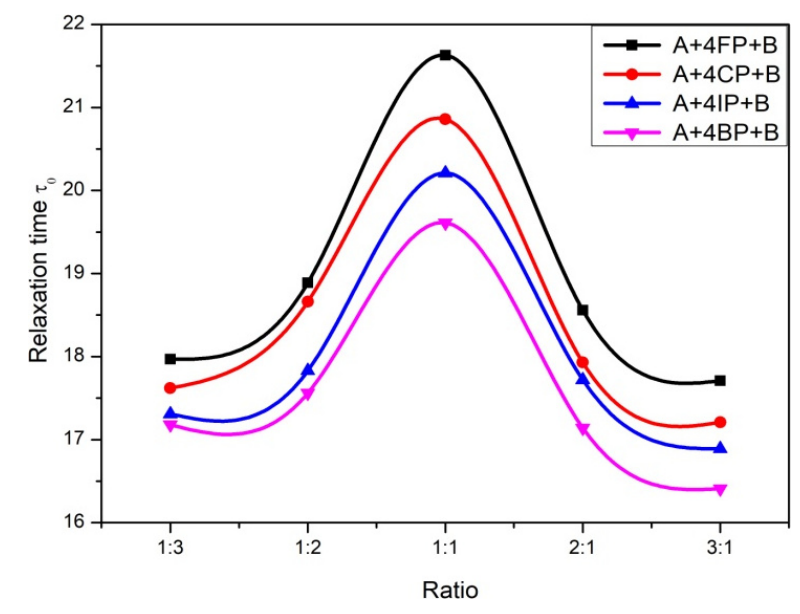

(b)

FIGURE 1. Variation of mean relaxation time vs. ratio of halogenated phenols with (a) benzamide and (b) acetamide in benzene. 


$$
\epsilon^{\prime}=\epsilon_{1}^{\prime}+a^{\prime} w_{2}
$$

$$
\epsilon_{\infty}=\epsilon_{\infty 1}+a_{\infty} w_{2}
$$

The equations of Eyring (1936) were used to quantify the free energy of activation for dielectric relaxation $\Delta F \tau$ and viscous flow $\Delta F \eta$ (Equations 7 and 8).

$$
\begin{gathered}
\tau=\frac{h}{k T} \exp \left(\frac{\Delta F_{\tau}}{R T}\right) \\
\eta=\frac{N h}{V} \exp \left(\frac{\Delta F_{\eta}}{R T}\right)
\end{gathered}
$$

\section{RESULTS AND DISCUSSION}

Benzamide and acetamide were chosen as proton donors of halogenated phenols like (4-fluorophenol, 4-bromophenol, 4-chlorophenol, and 4-iodophenol) in a ternary system with benzene as the solvent. The relaxation times $\tau_{(1)}$, $\tau_{(2)}$, and $\tau_{(0)}$ for both processes were calculated using Higasi's method (Saxena and Saxena 1981). In benzene, tables 1 and 2 show the relaxation time of amides with proton donors (4-fluorophenol, 4-bromophenol, 4-chlorophenol, and 4-iodophenol) at $35^{\circ} \mathrm{C}$. The relevance of relaxing period rises with increasing amide chain length and acidity disposition of phenolic compounds, according to a table. As a result, it is possible to pair the positive hydrogen of the alcohol group with the carbon atom in benzene. The benzene molecule's possible hydrogen bonds can contribute to a longer relaxation time.

Our consequences of Higasi show that it diminishes with diminishing amide fixation. This may be seen from the perspective of reverberation structures, which help to modify the stable base of electron - deficient amides. The free rotation of $\mathrm{C}=\mathrm{O}$ and $\mathrm{N}-\mathrm{H}$ in formamide and acetamide must be restricted due to this resonance structure by means of Thompson and Laplanche (Thompson and LaPlanche 1963). As the ratio of the electron - deficient amide was decreased gradually, the hydrogen bonded complexes stabilized by splitting in resonance form. This mechanism is aided by the solvent action of benzene, which has a higher Hydrogen bonding capacity with phenols than the amides own identity Hydrogen bonding capability. Similarly in our result show the same benzamide is a more efficient proton donor than acetamide, and it is more soluble in benzene. This is why benzamide has a longer relaxation time in benzene than acetamide at the same concentration. Amides basicity is determined by the substituent linked to the carbonyl group. When the benzene group bonds with the carbonyl group, the resonance is extended. As a result,

\begin{tabular}{|c|c|c|c|c|c|c|c|c|c|c|}
\hline \multirow[b]{2}{*}{ Ratio } & \multirow[b]{2}{*}{ Weight fraction $\left(\mathrm{W}_{2}\right)$} & \multirow[b]{2}{*}{$\epsilon_{0}$} & \multirow[b]{2}{*}{$\epsilon^{\prime}$} & \multirow[b]{2}{*}{$\epsilon^{\prime \prime}$} & \multirow[b]{2}{*}{$\epsilon_{\infty}$} & \multicolumn{3}{|c|}{ Relaxation time (ps) } & \multicolumn{2}{|c|}{ Activation energy $\left(\mathrm{KJ} \mathrm{mol}^{-1}\right)$} \\
\hline & & & & & & $\tau_{1}$ & $\tau_{2}$ & $\tau_{0}$ & $\Delta f \tau$ & $\Delta f \eta$ \\
\hline \multicolumn{11}{|c|}{ Benzamide+4FP+Benzene } \\
\hline $1: 3$ & 0.03839 & 6.5724 & 2.8913 & 0.8967 & 2.6008 & 20.91 & 21.10 & 21.01 & 29.82 & 29.88 \\
\hline $1: 2$ & 0.03874 & 6.5854 & 2.8869 & 0.8794 & 2.6024 & 22.32 & 21.58 & 21.95 & 29.95 & 30.08 \\
\hline $1: 1$ & 0.04015 & 6.6379 & 2.8740 & 0.8678 & 2.6097 & 23.77 & 22.88 & 23.32 & 30.74 & 30.98 \\
\hline $2: 1$ & 0.04011 & 6.6243 & 2.8856 & 0.8901 & 2.6088 & 22.35 & 21.61 & 21.90 & 29.99 & 30.10 \\
\hline $3: 1$ & 0.04046 & 6.5905 & 2.8999 & 0.9002 & 2.6087 & 20.94 & 21.43 & 21.18 & 29.88 & 29.87 \\
\hline \multicolumn{11}{|c|}{ Benzamide+4CP+Benzene } \\
\hline $1: 3$ & 0.04562 & 6.6401 & 2.9232 & 0.9253 & 2.6012 & 20.62 & 20.90 & 20.76 & 29.51 & 29.67 \\
\hline $1: 2$ & 0.04558 & 6.6503 & 2.9102 & 0.9044 & 2.6033 & 21.16 & 21.43 & 21.39 & 29.74 & 29.88 \\
\hline $1: 1$ & 0.04456 & 6.6795 & 2.8965 & 0.8732 & 2.6145 & 21.99 & 22.58 & 22.28 & 30.55 & 30.74 \\
\hline $2: 1$ & 0.04353 & 6.6975 & 2.9113 & 0.8950 & 2.6095 & 21.17 & 21.47 & 21.32 & 29.77 & 29.90 \\
\hline $3: 1$ & 0.04302 & 6.7101 & 2.9206 & 0.9116 & 2.6089 & 19.65 & 20.91 & 20.28 & 29.55 & 29.69 \\
\hline \multicolumn{11}{|c|}{ Benzamide+4IP+Benzene } \\
\hline $1: 3$ & 0.04339 & 6.7203 & 2.9504 & 0.9499 & 2.6054 & 19.46 & 20.75 & 20.11 & 29.26 & 29.39 \\
\hline $1: 2$ & 0.04311 & 6.7337 & 2.9318 & 0.9295 & 2.6107 & 20.37 & 21.29 & 20.83 & 29.48 & 29.59 \\
\hline $1: 1$ & 0.04275 & 6.7517 & 2.9211 & 0.8957 & 2.6202 & 21.69 & 22.76 & 22.23 & 30.14 & 30.43 \\
\hline $2: 1$ & 0.04233 & 6.7710 & 2.9307 & 0.9212 & 2.6105 & 20.97 & 21.33 & 21.15 & 29.51 & 29.63 \\
\hline $3: 1$ & 0.04212 & 6.7975 & 2.9342 & 0.9360 & 2.6102 & 20.12 & 20.13 & 20.19 & 29.29 & 29.38 \\
\hline \multicolumn{11}{|c|}{ Benzamide+4BP+Benzene } \\
\hline $1: 3$ & 0.05481 & 6.8021 & 2.9621 & 0.9653 & 2.6093 & 18.12 & 20.57 & 19.34 & 28.95 & 29.13 \\
\hline $1: 2$ & 0.05332 & 6.8175 & 2.9582 & 0.9445 & 2.6099 & 19.85 & 21.02 & 20.76 & 29.14 & 29.35 \\
\hline $1: 1$ & 0.05036 & 6.8308 & 2.9455 & 0.9347 & 2.6244 & 20.46 & 21.67 & 21.06 & 29.79 & 29.99 \\
\hline $2: 1$ & 0.04741 & 6.8558 & 2.9511 & 0.9405 & 2.6133 & 19.83 & 21.08 & 20.45 & 29.17 & 29.38 \\
\hline $3: 1$ & 0.04593 & 6.8753 & 2.9651 & 0.9682 & 2.6141 & 18.16 & 20.58 & 19.37 & 28.98 & 29.17 \\
\hline
\end{tabular}
the lone pairs of $\mathrm{N}$ are accessible for protonation. As a result, benzamide is more basic than acetamide.

TABLE 1. Value of dielectric constants and relaxation times for various weight fractions, benzamide with halogenated phenols in benzene. 


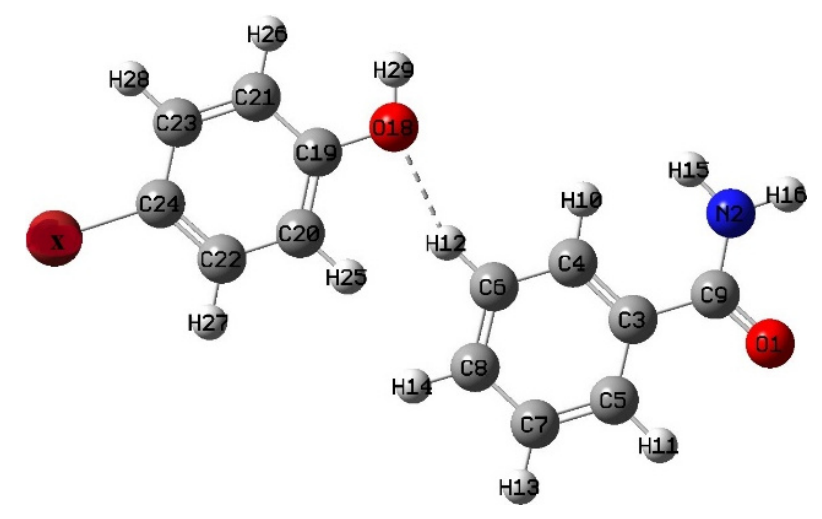

FIGURE 2. Formation of H-bonding between amide with halogenated phenols.

H-bonding in pyridine/phenol and quinoline/phenol structures now various conformations were investigated by various authors. They found that tertiary mixtures have a significantly longer relaxation time than all of the polarity substances throughout the neutral solution. Furthermore, if the complexes are tight, the distributed parameters are larger, and the two relaxation periods are well separated, one reflecting the complex's overall rotation and another indicating the rotations of several of the connecting polarized intermolecular forces. In the current research, the relaxation period for concentrated samples of p-cresol, p-chlorophenol, 2, 4-dichlorophenol, and pnitrophenol ranged from 4.5 to $34 \mathrm{ps}$. The relaxation times in amide/phenol systems are slightly longer because there are so many phenols present. This finding is consistent with Tucker and Walker (1966) and Basha et al. (2021), who studied the H-bonding in some carbonyl + phenols system in different compositions. These findings also suggest that the structural interaction among amides and phenolic compounds peaks at 1:1 mol\% and thereafter falls at other mol\% (Tables 1 and 2). As a consequence, we may deduce that the 1:1 complex is prevalent in amide/phenol complexes. In complex structures, the relaxation time $\tau_{0}$ rises as the sharpness of the proton donor rises, but due to steric effects and the stabilisation of complex compounds, the $\tau_{0}$ values of amides of 4-fluorophenol systems are greater than the other phenols complexes, as shown in Figure 1a and $1 \mathrm{~b}$.

Experimental acidic strength order: 4-bromophenol > 4-iodophenol $>4$-chlorophenol $>4$-fluorophenol. The $+\mathrm{R}$ effect of FF on 4-fluorophenol is considerable but weaker than the -I effect. Because of the stronger, the electron pair on the $2 p 2 p$ orbitals of FF may rapidly delocalize with the benzene ring, making the $+\mathrm{R}+\mathrm{R}$ effect strong. As a result, it improves the electron density on the carbon near the $-\mathrm{OH}$ -OH group to such an extent that it reduces its capacity to transfer protons, resulting in a significant drop in acidity. The $+\mathrm{R}$ effect is reduced for 4-chlorophenol because it is considerably weaker, and so the $-\mathrm{I}-\mathrm{I}$ effect becomes more significant. As a result, it becomes more difficult to remove hydrogen atoms from the $-\mathrm{OH}-\mathrm{OH}$ group, lowering its acidity.Because 4-bromophenol and 4-iodophenol

TABLE 2. Value of dielectric constants and relaxation times for various weight fractions, acetamide with halogenated phenols in benzene.

\begin{tabular}{|c|c|c|c|c|c|c|c|c|c|c|}
\hline \multirow[b]{2}{*}{ Ratio } & \multirow[b]{2}{*}{ Weight fraction $\left(W_{2}\right)$} & \multirow[b]{2}{*}{$\epsilon_{0}$} & \multirow[b]{2}{*}{$\epsilon^{\prime}$} & \multirow[b]{2}{*}{$\epsilon^{\prime \prime}$} & \multirow[b]{2}{*}{$\epsilon_{\infty}$} & \multicolumn{3}{|c|}{ Relaxation time (ps) } & \multicolumn{2}{|c|}{ Activation energy $\left(\mathrm{KJ} \mathrm{mol}^{-1}\right)$} \\
\hline & & & & & & $\tau_{1}$ & $\tau_{2}$ & $\tau_{0}$ & $\Delta f \tau$ & $\Delta f \eta$ \\
\hline \multicolumn{11}{|c|}{ Acetamide+4FP+Benzene } \\
\hline $1: 3$ & 0.0331 & 5.5231 & 2.8421 & 0.8421 & 2.5081 & 17.53 & 17.73 & 17.97 & 26.01 & 26.23 \\
\hline $1: 2$ & 0.0316 & 5.5437 & 2.8326 & 0.8262 & 2.5132 & 17.87 & 19.44 & 18.89 & 26.39 & 26.63 \\
\hline $1: 1$ & 0.0288 & 5.5642 & 2.8255 & 0.8648 & 2.5231 & 21.44 & 21.75 & 21.63 & 26.68 & 26.96 \\
\hline $2: 1$ & 0.0259 & 5.5745 & 2.8328 & 0.8369 & 2.5134 & 18.53 & 18.58 & 18.56 & 26.47 & 26.69 \\
\hline $3: 1$ & 0.0245 & 5.5979 & 2.8432 & 0.8238 & 2.5072 & 17.86 & 16.77 & 17.71 & 26.08 & 26.26 \\
\hline \multicolumn{11}{|c|}{ Acetamide+4CP+Benzene } \\
\hline $1: 3$ & 0.0408 & 5.6043 & 2.8571 & 0.8532 & 2.5092 & 17.44 & 17.82 & 17.62 & 25.39 & 25.79 \\
\hline $1: 2$ & 0.0385 & 5.6132 & 2.8435 & 0.8372 & 2.5152 & 17.82 & 19.66 & 18.66 & 25.57 & 26.02 \\
\hline $1: 1$ & 0.0274 & 5.6395 & 2.8302 & 0.8759 & 2.5254 & 20.69 & 20.98 & 20.86 & 26.06 & 26.82 \\
\hline $2: 1$ & 0.0293 & 5.6575 & 2.8416 & 0.8464 & 2.5144 & 17.97 & 17.88 & 17.93 & 25.69 & 26.09 \\
\hline $3: 1$ & 0.0255 & 5.6701 & 2.8493 & 0.8362 & 2.5089 & 17.92 & 16.83 & 17.21 & 25.52 & 25.84 \\
\hline \multicolumn{11}{|c|}{ Acetamide+4IP+Benzene } \\
\hline $1: 3$ & 0.0381 & 5.6903 & 2.8608 & 0.8671 & 2.5132 & 17.74 & 16.68 & 17.31 & 24.72 & 25.02 \\
\hline $1: 2$ & 0.0361 & 5.7037 & 2.8581 & 0.8467 & 2.5163 & 16.84 & 18.81 & 17.83 & 25.07 & 25.58 \\
\hline $1: 1$ & 0.0321 & 5.7117 & 2.8402 & 0.8852 & 2.5268 & 20.76 & 19.64 & 20.21 & 25.67 & 25.96 \\
\hline $2: 1$ & 0.0281 & 5.7113 & 2.8584 & 0.8526 & 2.5158 & 17.9 & 17.54 & 17.72 & 25.58 & 25.91 \\
\hline $3: 1$ & 0.0262 & 5.7154 & 2.8628 & 0.8462 & 2.5112 & 17.86 & 15.91 & 16.89 & 24.79 & 25.08 \\
\hline \multicolumn{11}{|c|}{ Acetamide+4BP+Benzene } \\
\hline $1: 3$ & 0.0495 & 5.7281 & 2.8759 & 0.8822 & 2.5168 & 17.62 & 16.73 & 17.18 & 24.02 & 24.29 \\
\hline $1: 2$ & 0.0462 & 5.7297 & 2.8658 & 0.8656 & 2.5175 & 16.18 & 18.93 & 17.56 & 24.21 & 24.38 \\
\hline $1: 1$ & 0.0397 & 5.7314 & 2.8557 & 0.8971 & 2.5283 & 19.68 & 19.53 & 19.61 & 24.54 & 24.82 \\
\hline $2: 1$ & 0.0300 & 5.7258 & 2.8657 & 0.8688 & 2.5179 & 17.71 & 16.56 & 17.14 & 24.24 & 24.41 \\
\hline 3:1 & 0.0321 & 5.7241 & 2.8754 & 0.8785 & 2.5153 & 16.94 & 15.88 & 16.41 & 24.19 & 24.35 \\
\hline
\end{tabular}


are substantially less electronegative, there is no strong hydrogen bonding, and the $+\mathrm{R}$ effect is ineffective due to its weakness. Only the -I effect fails in favour of acidity for these two compounds. As a result, 4-bromophenol has a higher acidity than 4-iodophenol.

The increasing relaxation time is due to the increasing chain length of phenols and amides, which creates a hindrance to molecule rotation. The increased relaxation time might be attributed to the spinning unit's current range increasing. When there is a high concentration of phenols in the mixture, the amides molecule is confined by a significant number of phenols molecules. As a result of these dipole-dipole interactions, the average charge distribution increases and linear multimers form. The relations of the -OH group of phenols through the $\mathrm{C}=\mathrm{O}$ of amides is known as dipole-dipole interactions. Only a small number of phenols molecules are required to allow dipole-dipole interactions by hydrogen bonding with independents of amides molecules at low concentrations of phenols in mixtures (Sivagurunathan et al. 2005; Shinde et al. 2019).

Ab intio molecular orbital calculations confirmed that the most stable conformers is gauche, stabilized by formation of inter (or) intramolecular hydrogen bond between the two hydroxyl groups above these systems. Thus only one hydroxyl group 4FP, 4BP, 4CP, and 4IP can interact with neighbouring molecule through $\mathrm{H}$-bond, the conformation of the $4 \mathrm{FP}, 4 \mathrm{BP}, 4 \mathrm{CP}$, and $4 \mathrm{IP}$ the chain length of $\mathrm{C}=\mathrm{O}$ group in benzene solution with more dilution. The gauche conformation of the $\mathrm{OCH} 2$ - $\mathrm{CH} 2 \mathrm{O}$ group predominantly stabilized. Furthermore there exists a specific interaction between X (4-fluorophenol, 4-chlorophenol, 4-bromophenol, and 4-iodophenol) and it has been suggested that the oxygen - oxygen distance is closely related to the interaction. This favours the formation of an gauche conformer of $\mathrm{OCH} 2$ - $\mathrm{CH} 2 \mathrm{O}$ group of $4 \mathrm{FP}, 4 \mathrm{BP}, 4 \mathrm{CP}$, and $4 \mathrm{IP}$ molecules with $\mathrm{C}=\mathrm{O}$ group in benzene the formation of intermolecular (or) intramolecular H-bonds (Kumar et al. 2011), as shown in Figure 2.

The activation molar free energy for viscous flow as evidenced by our findings, $\Delta F \eta$ is bigger than $\Delta F \tau$, the free energy of activation for dielectric relaxation. This is in accordance with the finding that viscous flow has larger dielectric relaxation values, as the previous phenomena happens exclusively through rotation, but viscous flow includes both longitudinal and transverse movements. It is also proposed that the free energy of activation for oscillation and viscous flow for regret polar liquids will be $\Delta F \tau$ is $<0.5 \Delta F \eta$ (Shirke et al. 2000; Thenappan and Sankar 2006). The molar free energy of activation for viscous flow $\Delta F \eta$ and the free energy $\Delta F \tau$ have been measured but also provided in Tables 1 and 2 for benzamide and acetamide with halogenated phenols (4-fluorophenol, 4-bromophenol, 4chlorophenol, and 4-iodophenol) using benzene.

These are consistent with evidence that viscous flow, that also includes both longitudinal and transverse movements, was subjected to more interaction from its surroundings unlike dielectric relaxation, which only occurs through oscillation (Chaudhari et al. 2002; Coelho et al. 2013). According to Smyth (Ratajczak and Orville-Thomas 1980), a proton donor relaxation time increases as the solute system acceptor capacity increases. A proton acceptor relaxation time must also increase in proportion to the donor solute proton donor potential. This study is inspired by our findings.

\section{CONCLUSIONS}

Acetamide and benzamide hydrogen bonded complexes with halogenated phenols like (4-fluorophenol, 4-bromophenol, 4-chlorophenol, and 4-iodophenol) have been found to be suitable for dilute benzene solutions using the dielectric method. At 1:1 molar ratios of amides to halogenated phenol, the values of average relaxation time $\tau_{(1)}$, total dielectric relaxation $\tau_{(2)}$ and mean relaxation time $\tau_{(0)}$ were found to be maximum. The length of the alkyl chain in phenols and amides affects the dielectric properties of the above systems. The most likely association between phenols and amide is 1:1 complex through the free hydroxyl group of the phenols and the carbonyl group of amides. From the above result it may be conclude that, the proton accepting ability of amides is in decreasing order: benzamide < acetamide.

\section{ACKNOWLEDGMENTS}

The authors thank the Management of The Islamiah College (Autonomous), for the facilities provided and the encouragements shown towards this work. We also thank our guide, Dr. F. Liakath Ali Khan and colleagues Dr. Muzammil P. and Mr. Fasiuddin G. S. of the Physics Department for their help.

\section{AUTHORS' CONTRIBUTIONS}

$\mathrm{AAB}$ contributed reagents, materials, and analysis tools. $\mathrm{AAB}$ and FLAK drafted the manuscript. All authors read and approved the final version of the manuscript.

\section{COMPETING INTERESTS}

The authors declare that there is no conflict or competing interests regarding the publication of this manuscript.

\section{REFERENCES}

Basha AA, Khan FLA, Muthu S. 2021. Dielectric studies of hydrogen bonded complexes of $\mathrm{C}=\mathrm{O}$ compounds with substituted phenols using frequency domain technique. J Adv Sci Res. 12(2 Suppl 1):180-185. https://sciensage. info/admin/uploads/paper/12\%20(2-1)132211.pdf.

Bone S, Pethig R. 1985. Dielectric studies of protein hydration and hydration-induced flexibility. J Mol Biol. 181(2):323-326. doi:10.3109/15368378509033258.

Chaudhari A, Ahire S, Mehrotrac S. 2001. Dielectric relaxation study of pyridine-water and pyridine-sulfolane mixtures using time domain reflectometry. J Mol Liq. 94(1):17-25. doi:10.1016/S0167-7322(01)00238-0.

Chaudhari A, Shirke RM, Nore NM, Patil PB. 2002. Dielectric study of n-butyl acetate-alcohol mixtures by timedomain reflectometry. J Solution Chem. 31(4):305-315. doi:10.1023/A:1015805304781.

Coelho JP, Naydenov GP, Yankov DS, Stateva RP. 2013. Experimental measurements and correlation of the solubility of three primary amides in supercritical $\mathrm{Co}_{2}$ : acetanilide, propanamide, and butanamide. J Chem Eng Data. 58(7):2110-2115. doi:10.1021/je400357t.

Eyring H. 1936. Viscosity, plasticity, and diffusion as examples of absolute reaction rates. J Chem Phys. 4(4):283291.

Gioia AL, Porter E, Merunka I, Shahzad A, Salahuddin S, Jones M, O'Halloran M. 2018. Open-ended coaxial 
probe technique for dielectric measurement of biological tissues: challenges and common practices. Diagnostics. 8(2):40. doi:10.3390/diagnostics8020040.

Grant EH, Sheppard RJ, South GP. 1978. Dielectric behaviour of biological molecules in solution. Clarendon Press.

Higasi K. 1966. Dielectric relaxation and molecular structure. II. Notes on analysing dielectric data of dilute solutions. Bull Chem Soc Jpn. 39(10):2157-2160. doi: 10.1246/bcsj.39.2157.

Jeffrey GA, Saenger W. 1991. Hydrogen bonding in biological structures. Springer. doi:10.1007/978-3-642-85135-3.

Kumar R, Baskar AJA, Kannappan V, RoopSingh D. 2014. Acoustical and spectroscopic investigation of charge transfer complexes of certain aromatic compounds with iodine in n-hexane at $303 \mathrm{~K}$. J Mol Liq. 196:404410. doi:10.1016/j.molliq.2014.03.035.

Kumar S, Periyasamy P, Jeevanandham P. 2011. Dielectric relaxation studies of binary liquid mixtures of a few glycols with 1, 4-dioxane. Int J ChemTech Res. 3(1):369375.

Kumar S, Sivakumar S, Jeevanandham P. 2010. Molecular interaction and dielectric relaxation studies of cycloalcohols with acetamide in a non-polar solvent using frequency domain technique. Int J Recent Sci Res. 7:177181.

Malathi M, Sabesan R, Krishnan S. 2003. Dielectric relaxation studies of dilute solutions of amides. Mater Sci Eng B. 104(1-2):1-4. doi:10.1016/S0921-5107(03)00141-7.

Rangra VS, Sharma DR. 2004. Dielectric relaxation studies of binary mixtures of acetone and n-methylacetamide in benzene solution using microwave absorption data. Indian J Pure Appl Phys. 2004(12):921-925.

Ratajczak H, Orville-Thomas WJ. 1980. Molecular interactions. volume 2. Wiley \& Sons.

Rewar GD, Bhatnagar D. 2002. Dielectric relaxation in ternary mixtures of benzotrifluorides. Indian J Pure Appl Phys. 40(6):430-435.

Saxena SK, Saxena MC. 1981. Dielectric relaxation in OH-O bond complexes. Ind J Pure Appl Phys. 19(6):550-554.

Sengwa R. 1994. Microwave dielectric relaxation studies of some ethylene oxide condensation products in pure liquid state. J Mol Liq. 62(1-3):139-146. doi:10.1016/0167-7 322(94)00771-3.

Shinde RV, Deshmukh AR, Ingole SA, Kumbharkhane AC. 2019. Dielectric spectroscopy and hydrogen bonding studies of 1-chloropropane-ethanol mixture using TDR technique. J Adv Dielectr. 09(02):1950018. doi:10.1142/ S2010135X19500188.

Shirke RM, Chaudhari A, More NM, Patil PB. 2000. Dielectric measurements on methyl acetate + alcohol mixtures at $(288,298,308$, and 318) $\mathrm{K}$ using the time domain technique. J Chem Eng Data. 45(5):917-919. doi: 10.1021/je000066.

Sisodia ML, Raguvanshi GS. 2005. Basic microwave techniques and laboratory manual. New Delhi: New Age International.

Sivagurunathan P, Dharmalingam K, Ramachandran K, Undre BP, Khirade PW, Mehrotra SC. 2005. Dielectric study of methyl methacrylate-alcohol mixtures by Time Domain Reflectometry at 293K. Main Group Chem. 4(3):235-240. doi:10.1080/10241220600628640.

Sivagurunathan P, Vijayabalan R. 2010. Molecular interaction on benzyl alcohol with formamides in carbon tetrachloride-dielectric relaxation studies. Int J Curr Res. 2:29-35.

Srinivasan N, Kumar S, Krishnan S. 2003. Solvent effect on linear correlation factor and dielectric relaxation time of butyl alcohol. Indian J Pure Appl Phys. 41(11):876-878.

Tabassum S, Pawar VP. 2018. Complex permittivity spectra of binary polar liquids using time domain reflectometry. J Adv Dielectr. 8(3):1850019. doi:10.1142/S2010135X185 00194.

Thakur N, Sharma DR. 2000. Dielectric relaxation of binary mixtures of acetonitrile and $\mathrm{N}, \mathrm{N}$-dimethyl formamide in benzene solution using microwave absorption studies. Indian J Pure Appl Phys. 38(05):328-330.

Thenappan T, Sankar U. 2006. Dielectric studies of hydrogen bonded complexes of alcohols with NN-dimethyl formamide. J Mol Liq. 126(1-3):38-42. doi:10.1016/j.mo lliq.2005.02.003.

Thompson HB, LaPlanche LA. 1963. Electric moment of N,Ndisubstituted amides ${ }^{1}$. J Phys Chem. 67(10):2230-2231. doi:10.1021/j100804a506.

Tucker SW, Walker S. 1966. Dielectric studies. part 10.relaxation of two intermolecularly hydrogen bonded complexes. Trans Faraday Soc. 62(0):2690-2694. doi: 10.1039/TF9666202690.

Vyas AD, Rana VA. 2002. Dielectric relaxation of some rigid polar molecules and their binary mixture in benzene solution. Indian J Pure Appl Phys. 40(01):69-71. 